Journal of Molecular Genetics 11 (1): 1-13, 2019

ISSN: $2070-4267$

(C) Medwell Journals, 2019

\title{
Recombinant DNA Technology and its Applications
}

\author{
Alemayehu Choramo and Motuma Debelo \\ Department of Veterinary Medicine, College of Agriculture and Veterinary Medicine, \\ Jimma University, Jimma, Ethiopia
}

\begin{abstract}
Human beings want enjoying highest attainable standard of basic health without discrimination of race, religion and political belief, economic or social condition. The benefits of medical, psychological and related knowledge have to be extended all people to the fullest attainment of health. Health is a state of complete physical, mental and social well-being and not merely the absence of disease or infirmity. Three factors affect human life greatly by: deficiency of food, health problems and environmental issues. Beside a clean and safe environment, food and health are basic human requirements. Humans want safe-food at reasonable price. Numerous human related health issues across the world cause huge number of deaths. Therefore, the objective of this study is to review techniques and benefits of recombinant DNA technology. Recombinant DNA technology is an important development in science that has made the human life much easier. Two major categories of enzymes are important tools in the isolation of DNA and the preparation of recombinant DNA technique are restriction endonucleases and DNA ligases. Recombinant DNA techniques has seven steps (isolation of gene, preparation of target DNA insertion of DNA into vector insertion of plasmid back into cell, plasmid multiplication, target cell production and cells produced proteins). Cloning vectors such as YACS, bacteriophage, BACS, cosmids and plasmids are used for cloning of DNA. Recombinant DNA technology has numerous applications in agriculture, health and diseases and environmental applications. It is expected from, agricultural, medical and environmental sectors to work on this field, train professionals and introduce its uses to the society.
\end{abstract}

$\underline{\text { Key words: Safe-food, ezymes, technique, cells produced proteins, agriculture, society }}$

\section{INTRODUCTION}

Human being has the right of enjoying highest attainable standard of fundamental health without discrimination of race, religion and political belief, economic or social condition. The extension to all peoples of the benefits of medical, psychological and related knowledge is essential to the fullest attainment of health. Health is a state of complete physical, mental and social well-being and not merely the absence of disease or infirmity (Anonymous, 2018).

Human life is greatly affected by 3 factors: deficiency of food, health problems and environmental issues. Food and health are basic human requirements beside a clean and safe environment. With increasing world's population at a greater rate, human requirements for food are rapidly increasing. Humans require safe-food at reasonable price. Several human related health issues across the globe cause large number of deaths (Khan et al., 2016). Approximately, 36 million people die each year from non-communicable and communicable diseases such as cardiovascular diseases, cancer, diabetes, AIDS/HIV, tuberculosis, malaria and several others. Despite extensive efforts being made, the current world food production is much lower than human requirements and health facilities are even below standard in the third-world countries. Rapid increase in industrialization has soared up the environmental pollution and industrial wastes are directly allowed to mix with water which has affected aquatic marines and indirectly, human-beings (Kumar and Kumar, 2015).

Therefore, these issues urge to be addressed through modern technologies (Kumar and Kumar, 2015) such as recombinant DNA techniques. Recombinant DNA technology is an important development in science that has made the human life much easier. In recent years, it has advanced strategies for biomedical applications. The elucidation of the structure, function and metabolism of Deoxyribo Nucleic Acid (DNA) has led to the current global revolution in the recombinant DNA technology with the possibility to modify these molecules in many ways for the benefit of man and animals (Soetan and Abatan, 2008). Recombinant DNA technology is a fast growing field and researchers

Corresponding Author: Alemayehu Choramo, Department of Veterinary Medicine, College of Agriculture and Veterinary Medicine, Jimma University, Jimma, Ethiopia 
around the globe are developing new approaches, devices and engineered products for application in different sectors including agriculture, health and environment (Khan et al., 2016).

In the past century, the recombinant DNA technology was just an imagination that desirable characteristics can be improved in the living bodies by controlling the expressions of target genes. However, in recent era, this field has demonstrated unique impacts in bringing advancement in human life (Kumar and Kumar, 2015). Recombinant DNA technology offers a rational approach to the understanding of the molecular basis of a number of diseases, e.g., sickle cell disease, cystic fibrosis, etc. Human proteins can be produced in abundance for therapeutic purposes e.g., insulin, growth hormone, recombinant factor 8 , etc. Proteins for vaccines (e.g., Hepatitis B) and for diagnostic tests (e.g., AIDS Test) can be obtained. It's used to diagnose existing diseases and predict the risk of developing a given disease (Soetan and Abatan, 2008).

By virtue of this technology, crucial proteins required for health problems and dietary purposes can be produced safely, affordably and sufficiently. This technology has multidisciplinary applications and potential to deal with important aspects of life for instance, improving health, enhancing food resources and resistance to divergent adverse environmental effects (Kumar and Kumar, 2015). Particularly in agriculture, the genetically modified plants have augmented resistance to harmful agents, enhanced product yield and shown increased adaptability for better survival. Moreover, recombinant pharmaceuticals are now being used confidently and rapidly attaining commercial approvals. Techniques of recombinant DNA technology, gene therapy and genetic modifications are also widely used for the purpose of bioremediation and treating serious diseases (Khan et al., 2016).

Recombinant DNA technology is playing a vital role in improving health conditions by developing new vaccines and pharmaceuticals. The treatment strategies are also improved by developing diagnostic kits, monitoring devices and new therapeutic approaches. Synthesis of synthetic human insulin and erythropoietin by genetically modified bacteria and production of new types of experimental mutant mice for research purposes are one of the leading examples of genetic engineering in health (Lomedico, 1982). Likewise, genetic engineering strategies have been employed to tackle the environmental issues such as converting wastes into biofuels and bioethanol cleaning the oil spills, carbon and other toxic wastes and detecting arsenic and other contaminants in drinking water (Ullah et al., 2015).
The genetically modified microbes are also effectively used in biomining and bioremediation. The advent of recombinant DNA technology revolutionized the development in biology and led to a series of dramatic changes (Khan et al., 2016). It offered new opportunities for innovations to produce a wide range of therapeutic products with immediate effect in the medical genetics and biomedicine by modifying microorganisms, animals and plants to yield medically useful substances (Galambos and Sturchio, 1998). Most biotechnology pharmaceuticals are recombinant in nature which plays a key role against human lethal diseases (Khan et al., 2016). The pharmaceutical products synthesized through recombinant DNA technology (Galambos and Sturchio, 1998), completely changed the human life in such a way that the US Food and Drug Administration (FDA) approved more recombinant drugs in 1997 than in the previous several years combined which includes anemia, AIDS, cancers, hereditary disorders, diabetic foot ulcers, diphtheria, genital warts, Hepatitis B and C, human growth hormone deficiency and multiple sclerosis. Considering the plants develop multi-gene transfer, site specific integration and specifically regulated gene expression are crucial advanced approaches (Khan et al., 2016). Transcriptional regulation of endogenous genes, their effectiveness in the new locations and the precise control of transgene expression are major challenges in plant biotechnology which need further developments for them to be used successfully (Liu et al., 2013). Genetic engineering has replaced the conventional strategies and has the greater potential to overcome such challenges (Khan et al., 2016). Therefore, the objectives of this study are: to review tools, techniques and the benefits of recombinant DNA technology.

\section{MATERIALS AND METHODS}

Enzymes for recombinant DNA techniques: Two major categories of enzymes are important tools in the isolation of DNA and the preparation of recombinant DNA technique are restriction endonucleases and DNA ligases. Restriction enzymes are DNA-cutting enzymes found in bacteria (Brown, 2006). Because they cut within the molecule, they are often called restriction endonucleases. In order to be able to sequence DNA it is first necessary to cut it into smaller fragments. Many DNA digesting enzymes can do this but most of them are of no use for sequence work because they cut each molecule randomly (Stryjewska et al., 2013). This produces a heterogeneous collection of fragments of varying sizes. What needed is a way that cleaves the DNA molecule at a few precisely located sites, so that, a small set of homogeneous 
fragments are produced. The tools for this are the restriction endonucleases. The rarer the site it recognizes, the smaller the number of pieces produced by a given restriction endonuclease (Brown, 2006). DNA ligase covalently links the 2 into a molecule of recombinant DNA. To be useful, the recombinant molecule must be replicated many times to provide material for analysis and sequencing (Shivanand and Noopur, 2010).

Restriction enzyme: Restriction enzyme or endonuclease is an enzyme that cuts double stranded DNA. Precisely, it cleaves the sugar-phosphate backbone of DNA without damaging the bases. A restriction endonuclease works by scanning the length of DNA molecule and once it encounters its particular specific recognition sequence; it will bind to the DNA and makes one incision in each of the two sugar-phosphate backbones of the double helix. Thus, rather than cutting DNA indiscriminately, a RE cuts only at a particular nucleotide sequence, termed as recognition sequence. Therefore, scientists could cut the DNA molecule in a predictable and reproducible way. Restriction enzymes are traditionally classified into 3 types on the basis of subunit composition, cleavage position, sequence specificity and requirement for cofactors.

Type 1 restriction enzymes: Complex, multi-subunit, cut DNA at random, far from their recognition sequences. They may have biological significance but have a very little practical value, since, they do not produce discrete restriction fragments.

Type 2 restriction enzymes: Cut DNA at very precise and defined positions close to or within the recognition sequences. It is the only class of restriction endonuclease used in the laboratory for DNA analysis and gene cloning. They require only magnesium for activity and corresponding model of enzymes require only S-Adenosyl
Methionine (SAM). They are smaller with subunits in the range of 200-350 amino acids. The most common are the ones which cleave within symmetric recognition sequence, e.g., tha 1 and Hind 3. Next common of type 2 , the ones which cleave outside their recognition sequence to 1 side. They are intermediate in size with 400-650 amino acids and recognize sequences that are continuous and asymmetric. Third major type 2 restriction enzymes are referred to as type 4 are large, $850-1250$ amino acids where 2 enzymatic activities reside in the same protein chain, cleave outside the recognition sequence and others those recognize discontinuous sequences and cleave on both sides releasing a small fragment containing recognition sequence (Khan et al., 2016).

Type 3 restriction enzymes: Large, cleave outside the recognition sequence and require 2 sequences in opposite orientations within the same DNA molecule to accomplish cleavage. They rarely give complete digests. They have basically no practical requirement and there is none, so, available commercially.

DNA ligase: Ligases are vital enzymes required for physiological cellular processes such as DNA replication, repair of damaged DNA and recombination. This enzyme facilitates formation of phosphodiester bonds between adjacent $3{ }^{\circ} \mathrm{OH}$ and 5' phosphate termini, thereby joining the nicks in double strandeded DNA. The enzyme can catalyze the joining of two large molecules by forming a new chemical bond, usually with the accompanying hydrolysis of a small chemical group that is dependent on one of the larger molecules or the enzyme catalyzing the linking together of two compounds fill the gaps incurred in the helix (Stryjewska et al., 2013). There are two categories of ligase depending on the requirement for cofactors. Eukaryotic and virally encoded enzymes are ATP dependent whereas most prokaryotic enzymes require $\mathrm{NAD}+$ for their activity as a cofactor (Fig. 1).

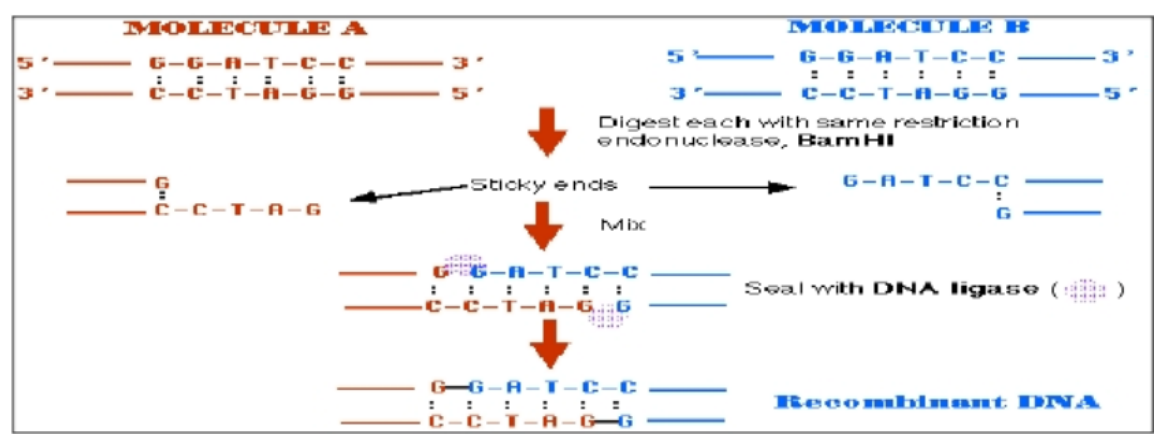

Fig. 1: Two DNA molecules from different source ligated with ligase enzyme (Shivanand and Noopur, 2010) 


\section{Cloning vectors}

Yeast Artificial Chromosome (YACs): These are linear DNA molecules similar to yeast chromosome. Recombinant YACs are made by ligating large fragments of genomic DNA and then the resultant plasmid is introduced into yeast by transformation (Stryjewska et al., 2013). The vector carries selection marker, DNA sequences called as telomere, so that, the product can be stabilized inside the yeast cell an origin of replication called Autonomous Replication origin, ARS. Large size of DNA can be inserted into YAC vectors, usually between $250-400 \mathrm{kbp}$. Large size of mammalian genomic libraries is also made with approximately 1 mega base pairs of Foreign inserts. Insertion of foreign DNA into the cloning site inactivates a mutant expressed in vector DNA and formation of red rather than white colonies by yeast strain is observed.

Bacteriophage Artificial Chromosomes (BACs): These plasmids are circular DNA molecules carrying conventional antibiotic resistance marker origin of replication derived from the $\mathrm{F}$ factor of $E$. coli an ATP driven helicase (repE) to facilitate DNA replication and 3 loci ( $\mathrm{A}, \mathrm{B}$ and par $\mathrm{C}$ ) for proper partitioning of the plasmid to daughter cells. BAC vectors have no packing constraints and there is no fixed limit to the size of genomic DNA that they accept. Usually, the size of DNA is approximately $120 \mathrm{kbps}$.

Cosmids: The cosmid vectors are opened up by appropriate restriction enzymes and then mixed with the DNA inserts prepared by using same enzymes under annealing conditions. Long concatemers, so, generated are products for packaging into the $\lambda$ heads. This selects the DNA to be inserted, since, for packaging in $\lambda$ particles, the distance between two cos sites must be between $38-52 \mathrm{~kb}$. Cosmids can accommodate up to $45 \mathrm{kbp}$ long DNA inserts. Cosmids infect the host cells like $\lambda$ particles but once inside the host it will replicate and propagate as a plasmid.

Bacteriophage vectors: A phage (also called bacteriophage) is a small virus that infects only bacteria. Like viruses that infect eukaryotes, phages consist of an outer protein coat and the enclosed genetic material (which consists of double-stranded DNA in $95 \%$ of the phages known and few have RNA as the genetic material) of 5-650 kbp (Kilo Base Pairs) with a length of 24-200 nm. Phages were discovered independently by Frederick Twort and Felixd' Herelle. Phages infect only specific bacteria. Some phages are virulent that is upon infecting a bacterial cell, they immediately start reproducing and within a short time, lyse (destroy) the bacterial cell, releasing new phage particles. These young phages burst from the host cell (killing it) and infect more bacteria. These types of phages are called as Lytic phages. Some phages (so-called temperate phages) can instead enter a relatively harmless state, either integrating their genetic material into the chromosomal DNA of the host bacterium (much like endogenous retrovirus in animals) or establishing themselves as plasmids.

Plasmids: The word "plasmid" was introduced by Joshua Lederberg and is defined as an extra-chromosomal circular DNA molecule which are not part of the bacterial genome. A plasmid is an independent, circular, self replicating DNA molecule that carries few genes only. Plasmids are usually dependent on host synthesized enzymes and proteins for their replication and transcription (Stryjewska et al., 2013). The three most widely studied types of plasmids are:

- F plasmids responsible for conjugation

- R plasmids carry genes for resistance to antibiotics

- Col plasmids code for colicins, the proteins that kill sensitive $E$. coli cells and also carry genes that provide immunity to the particular colicin

Vectors contain genetic markers that confer strong growth advantages under selective conditions. The plasmid DNA containing Foreign DNA as insert is usually introduced artificially by transformation. Selection is then carried out by screening for the colonies those have acquired the plasmid and hence, grow on Antibiotic containing plates. Routinely used antibiotics include ampicillin, kanamycin, chloramphenicol, carbenicillin and tetracycline.

Gene cloning: Gene cloning is the technique whereby multiple copies of a plasmid or other cloning vehicles are produced by inserting the plasmid into a suitable host capable of producing multiple copies and growing in a bulk culture. The bacterium Escherichia coli is often used as the host organism for this purpose. The word gene cloning is often used in place of genetic engineering. DNA cloning have been permitted the development of modern biology in particular in the last 40 years we have passed from theorize DNA cloning to clone a gene using fast techniques and widespread in all research laboratories, moreover, there are company that can clone any DNA fragment, coding or regulative at relatively low cost, these clones can be used to transform bacterial cells and transecting eukaryotic cells (Mandrich, 2013). 
Isolation of gene: In order to clone a gene the first step is to isolate it using restriction enzymes. These enzymes recognize specific regions on the DNA molecule (Stryjewska et al., 2013). The region of DNA shown below is from Rhodobacter sphaeroides. The gene of interest lies in the region of the chromosome indicated in blue. The base sequences are the ones that the restriction enzyme (EcoRI) recognizes. Note that reading from left to right in the top strand is the same as reading from right to left in the bottom strand. Use EcoRI to cut the sugar-phosphate backbone at the points indicated by the red arrows (Fig. 2-5).

The gene for producing a protein is isolated from a cell. The gene is on the DNA in a chromosome. Special DNA cutting proteins are used to cut out certain sections of DNA. The gene can be isolated and then copied, so that, many genes are available to work with Shivanand and Noopur (2010).

Unpaired bases result when EcoRI cuts a DNA molecule. Note that the gene of interest is bounded by fragments of DNA containing unpaired bases or "sticky ends". If the temperature is lowered and DNA ligase is added these unpaired bases can reanneal following the rules of base pairing (Brown, 2006).

Preparation of target DNA: Boyer and Cohen (1973) developed a way to put DNA from one organism into the DNA of bacteria. This process is called recombinant DNA technology. First, a circular piece of DNA called a plasmid is removed from a bacterial cell. Special proteins are used to cut the plasmid ring to open it up (Shivanand and Noopur, 2010) (Fig. 6 and 7).

Insertion of DNA into vector: The host DNA that produces the wanted protein is inserted into the opened plasmid DNA ring. Then special cell proteins help close the plasmid ring (Stryjewska et al., 2013).
Insertion of plasmid back into cell: The circular plasmid DNA that now contains the host gene is inserted back into a bacteria cell. The plasmid is a natural part of the bacteria cell. The bacteria cell now has a gene in it that is from a different organism, even from a human. This is what is called recombinant DNA technology (Shivanand and Noopur, 2010).

Plasmid multiplication: The plasmid inserted into the bacteria cell can multiply to make several copies of the wanted gene. Now the gene can be turned on in the cell to make proteins (Shivanand and Noopur, 2010).

Target cell production: Many recombined plasmids are inserted into many bacteria cells. While they live, the bacteria's cell processes turn on the inserted gene and the

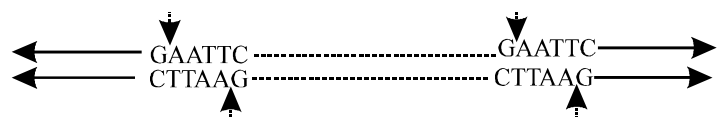

Fig. 2: Cutting DNA with restriction enzyme to isolate the required gene (Shivanand and Noopur, 2010)

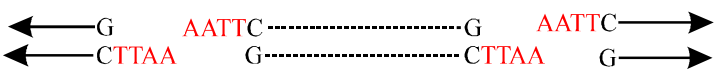

Fig. 3: Unpaired bases resulted when EcoRI cuts a DNA molecule

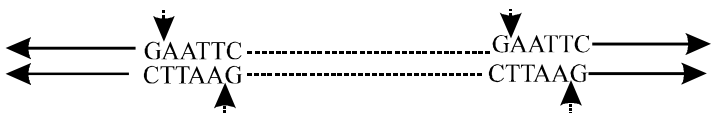

Fig. 4: Reanneling cut molecules when temperature is lowered

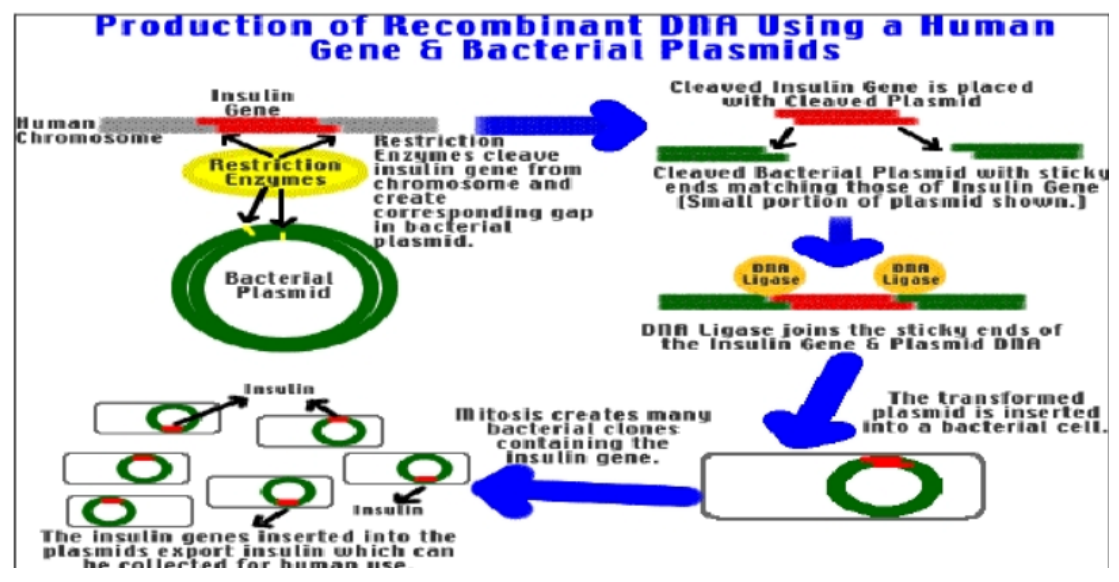

Fig. 5: Inserting DNA into vector (Shivanand and Noopur, 2010) 


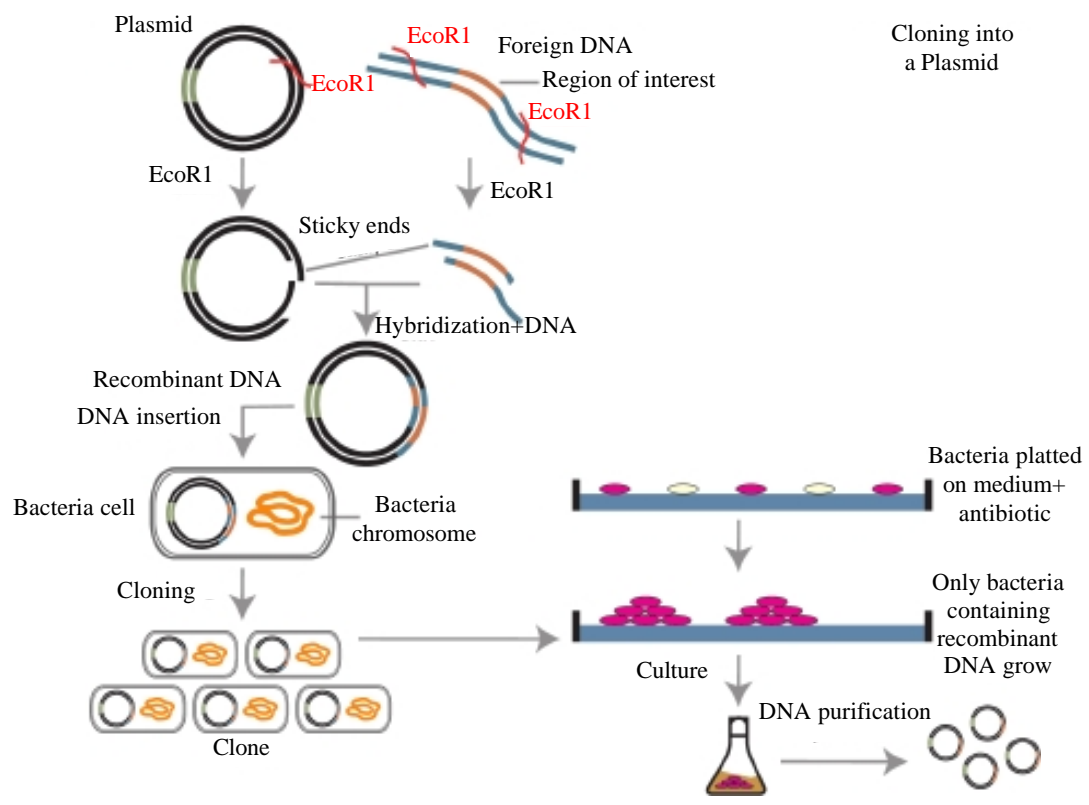

Fig. 6: Insertion of plasmid back into cell (Stryjewska et al., 2013)

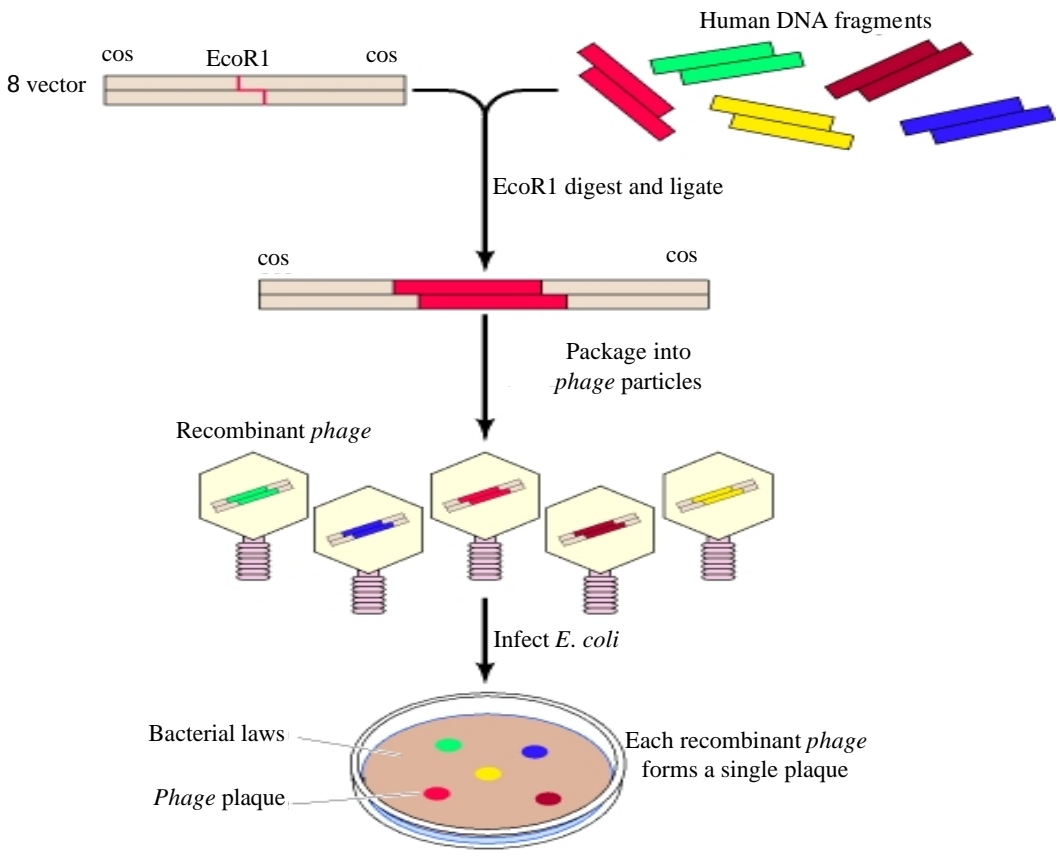

Fig. 7: Production of target cells

protein is produced in the cell. When the bacterial cells reproduce by dividing, the inserted gene is also reproduced in the newly created cells.

Cells produced proteins: The protein that is produced can be purified and used for a medicine, industrial, agricultural or other uses (Shivanand and Noopur, 2010).

\section{RESULTS AND DISCUSSION}

\section{Application of recombinant DNA technology}

Food and agriculture: Recombinant DNA technology has major uses which made the manufacturing of novel enzymes possible which are suitable in conditions for specified food processing. Several important enzymes 
including lipases and amylases are available for the specific productions because of their particular roles and applications in food industries (Khan et al., 2016). Microbial strains production is another huge achievement that became possible with the help of recombinant DNA technology. A number of microbial strains have been developed which produce enzyme through specific engineering for production of proteases. Certain strains of fungi have been modified, so that, their ability of producing toxic materials could be reduced (Olempska- Beer et al., 2006). Lysozymes are the effective agents to get rid of bacteria in food industries. They prevent the colonization of microbial organisms. It is suitable agent for food items including fruits, vegetables, cheese and meat to be stored as it increases their shelf life. The inhibition of food spoiling microorganisms can be carried out through immobilized lysozyme in polyvinyl alcohol films and cellulose (Khan et al., 2016). Lysozyme impregnation of fish skin gelatin gels increase the shelf life of food products and inhibit different food spoiling bacterial growth (Thallinger et al., 2013).

Plants have been used to produce several therapeutic protein products such as casein and lysozyme for improving health of child and polymers of protein for tissue replacement and surgery. Furthermore, tobacco plants can be engineered genetically to produce human collagen. High yielding molecular proteins is one of the major tasks under consideration in field of recombinant DNA technology (Ma et al., 2003). Traditional breeding and Quantitative Trait Locus (QTL) analysis assisted in the identification of a rice variety with protein kinase known as PSTOL1 (Phosphorus Starvation Tolerance 1) help in enhancing root growth in early stages and tolerates phosphorus deficiency. Over expression of this enzyme enables root to uptake nutrients in sufficient amount in phosphorus deficient soil which ultimately enhances the grain yield (Khan et al., 2016).

Resistance to fungal and bacterial infections can be enhanced by WRKY 45 gene in rice which is induced by plant activator benzothiadiazole that activates innate immune system of plant. The larger grain size can be achieved by inserting qSW5 gene. The qSH1 causes the loss of seed shattering by preventing the abscission layer formation. Kala 4 gene is responsible for the black color of rice which makes the rice resistant to attacking pathogens (Oikawa et al., 2015). Genetic modification is needed in facilitating gene by gene introduction of well-known characters. It allows access to extended range of genes from an organism. Potato, beans, eggplant, sugar beet, squash and many other plants are being developed with desirable characters, for example, tolerance of the herbicide glyphosate, resistance to insects, drought resistance, disease and salt tolerance. Nitrogen utilization, ripening and nutritional versatility like characters have also been enhanced (Khan et al., 2016).

Development of genetically modified crops with a purpose to improve both yield and resistance to plant pests or herbicides seems to have gained a degree of public acceptance and is already practiced in a commercial context in several countries. The genetically modified tomato CGN-89564-2 was the first commercially grown, genetically engineered crop product to be granted a licence for human consumption (Rajakaruna and Taylor-Robinson, 2016).

\section{Production of good quality and high yielding animal:} Livestock production is expected to grow tremendously in line with the projected demand for animal products. Therefore, the methods of livestock production must be changed to allow for efficiency and improvement in productivity. Biotechnological research is important in order to respond to the pressure of producing more food from animals to cater food requirement of the ever-growing human population (Kahi and Rewe, 2008). Transgenic animals such as mice, rats, rabbits, pigs, sheep and cows have been developed with the help of recombinant DNA technology. Transgenesis is the technique that permits the manipulation of genes of one organism which can subsequently be introduced into genome of another organism of same or other species in such a way that the genes are not only expressed but also gets transmitted to its progeny (Raghavan and Goswami, 2007).

Transgenic animals, thus, produced will have enhanced growth rate and improved food quality. For example, transgenic cows are developed to produce milk containing specific human proteins that helps in the treatment of human emphysema. Cloned transgenic cattle produced increased amount of beta and kappa casein in milk fat and increased level of human lacto-ferrin (Brophy et al., 2003). So, also such cows have been known to produce more milk or milk with less lactose or cholesterol, pigs and cattle that have more meat on them and sheep that yield more wool. Pigs with human IGF1 (Insulin like Growth Factor-1) had 30\% more loin mass, $10 \%$ more carcass lean tissue and $20 \%$ less total carcass fat (Pursel et al.,1999). Transgenic pigs carrying plant gene had increased amount of unsaturated fatty acids in their muscle to produce a meat called "Healthy Pork". Major genes for meat quality offer excellent opportunities for increasing level of meat quality and decreasing variability. Most scientists say that tenderness is $30 \%$ and Pale Soft Exudate (PSE) condition in swine is 50\% genetically governed characters. The quality of carcass 
can be improved by manipulating the lipoprotein receptor and leptin genes thereby the cholesterol and fat content of meat can be governed (Gupta and Savalia, 2012).

There is a growing database supporting the use of pituitary derived Somatotropin (ST) as an agent to improve growth and carcass composition. Pigs injected with pST had $35 \%$ less fat and $8 \%$ more protein. However, purification of ST from pituitary gland is uneconomical as production of single dose requires collection and processing of 25-100 pituitary glands. More recently, the development of recombinant DNA technology has provided a mechanism for large scale production of somatotropin (Gupta and Savalia, 2012). The gene for ST protein is inserted in laboratory strain of $E$. coli which can be grown on a large scale and from which ST can be purified and concentrated. Bovine and ovine ST improves growth rate by $20 \%$ and lean to fat ratio by $40 \%$ in ruminants. While experimentation in growing pigs, significant improvement of $40 \%$ in average daily gain and $30 \%$ in feed conversion efficiency had been achieved by administration of pST. Furthermore $60 \%$ reduction in carcass fat and $70 \%$ increase in carcass protein had been attained.

Byproduct utilization: A major concern in the food processing industry is the development of methods to convert inedible and waste materials into new value-added products. Environmental and economic concerns necessitate a reduction of food processing waste, better use of raw materials and by products to new value added products. A recently developed bioconversion system employing selected strains of yeast can convert these solids to ascorbic acid with a market value of about $\$ 10$ per $\mathrm{kg}$. The yeast biomass could be dried and used as single-cell protein supplements in animal feed. Enzymatic treatment of food processing waste stream scan produce materials readily metabolized by genetically engineered micro-organisms to produce antibiotics, hormones or peptides of interest in the pharmaceutical or chemical industries (Gupta and Savalia, 2012).

Health and diseases: Recombinant DNA technology is providing highly sensitive and specific tools for the diagnosis, prognosis and disease surveillance in humans and animals. In the future, preventive human and veterinary medicine will focus on genetically engineered animals resistant to specific endemic diseases. Gene's conferring resistance to specific diseases may be copied, sequenced and propagated using PCR and inserted into the genes of recipient humans and animals (Kwaga and Kabir, 1999). Recombinant DNA technology has wide spectrum of applications in treating diseases and improving health conditions (Khan et al., 2016). The following sections describe the important breakthroughs of recombinant DNA technology for the improvement of health.

Gene therapy: This is another application of gene cloning in medicine and veterinary medicine. Gene therapy is the name given to methods that aim to cure an inherited disease by providing the patient with a correct copy of the defective gene. Gene therapy is with a therapeutic potential in health services. A functioning gene is inserted into a cell to correct a metabolic abnormality or to introduce a new function is one of the outcomes of breakthroughs in molecular biology. Gene therapy is a promising approach to the treatment of cancer and other genetic diseases in human and animals (Soetan and Abatan, 2008). The first successful report in field of gene therapy to treat a genetic disease provided a more secure direction toward curing the dead list genetic diseases (www.researchgate.net). This strategy shows good response in providing treatment for Adenosine Deaminase-Deficiency (ADA-SCID) which is a primary immunodeficiency (Aiuti et al., 2002).

Many different cancers including lung, gynecological, skin, urological, neurological and gastrointestinal tumors as well as hematological malignancies and pediatric tumors have been targeted through gene therapy. Inserting tumor suppressor genes to immunotherapy, oncolytic virotherapy and gene directed enzyme pro-drug therapy are different strategies that have been used to treat different types of cancers (Khan et al., 2016). The p53, a commonly transferred tumor suppressor gene is a key player in cancer treating efforts. In some of the strategies, p53 gene transfer is combined with chemotherapy or radiotherapy. The most important strategies that have been employed until now are vaccination with tumor cells engineered to express immune-stimulatory molecules, vaccination with recombinant viral vectors encoding tumor antigens and vaccination with host cells engineered to express tumor antigens Cancer gene therapy has become more advanced and its efficacy has been improved in recent years (Ginn et al., 2013).

Treatment of cardiovascular diseases by gene therapy is an important strategy in health care science. In cardiovascular field, gene therapy will provide a new avenue for therapeutic angiogenesis, myocardial protection, regeneration and repair, prevention of restenosis following angioplasty, prevention of bypass graft failure and risk-factor management (Khan et al., 2016). 
Production of antibodies and their derivatives: Plant systems have been recently used for the expression and development of different antibodies and their derivatives. Most importantly, out of many antibodies and antibody derivatives, seven have reached to the satisfactory stages of requirements. Transgenic tobacco plants can be used for the production of chimeric secretory $\operatorname{Ig} \mathrm{A} / \mathrm{G}$ known as CaroRx (Khan et al., 2016). Oral pathogen responsible for decay of a tooth known as Streptococcus mutants can be recognized by this antibody. A monoclonal antibody called T84.66 can effectively function to recognize antigen carcinoembryonic which is still considered an affectively characterized marker in cancers of epithelia (Stoger et al., 2000). A full length humanized IgG1 known as anti-HSV and anti-RSV which can function as the recognizing agent for Herpes Simplex Virus (HSV)-2-glycoprotein B has been expressed in transgenic soybean and Chinese Hamster Ovary $(\mathrm{CHO})$ cells. Antibodies from both sources have been shown to prevent vaginal HSV-2 transmission in mice after applying topically; if worked similarly in humans it would be considered as inexpensive and affective prevention against diseases transmitted through sexual interactions (Ionescu et al., 2008).

Investigation of the drug metabolism: Complex system of drug metabolizing enzymes involved in the drug metabolism is crucial to be investigated for the proper efficacy and effects of drugs. Recombinant DNA approaches have recently contributed its role through heterologous expression where the enzyme's genetic information is expressed in vitro or in vivo through, the transfer of gene (Rostami-Hodjegan and Tucker, 2007).

Development of recombinant hormones: Comparatively conventional vaccines have lower efficacy and specificity than recombinant vaccine. A fear free and painless technique to transfer adenovirus vectors encoding pathogen antigens is through nasal transfer which is also a rapid and protection sustaining method against mucosal pathogens. This acts as a drug vaccine where an anti-influenza state can be induced through a transgene expression in the airway (Zhang et al., 2011). In vitro production of human Follicle-Stimulating Hormone (FSH) is now possible through recombinant DNA technology. FSH is considerably a complex heterodimeric protein and specified cell line from eukaryotes has been selected for its expression. Assisted reproduction treatment through stimulating follicular development is an achievement of recombinant DNA technology. A large number of patients are being treated through recombinant Follicle-Stimulating Hormone (r-FSH). Most interestingly
r-FSH and Luteinizing Hormone (LH) recombination was made successful to enhance the ovulation and pregnancy (Khan et al., 2016).

\section{Recombinant vaccine}

Viral vectors: Most viral vectors are developed using viruses that are associated with mild or no disease or using viruses that are pathogenic but attenuated by deletion of virulence genes. Replication competent virus vectors which can produce progeny virus as well as replication-defective virus vectors which do not produce progeny virus have been developed and evaluated as vaccine delivery vehicles. A number of commercial vaccines based on DNA virus vectors including poxviruses and herpes viruses have been successfully licensed for use in veterinary medicine. These include vectors based on vaccinia virus, canary pox virus, fowl pox virus and turkey herpes virus. A number of viral vectors have been developed or are in the process of being developed, improved and evaluated (Gerdts et al., 2006).

Gene-deleted vaccines: The knowledge of specific virulence factor (s) of a pathogen and the availability of recombinant DNA technology has facilitated the creation of specific gene-deleted pathogens for use as live vaccines (Meesun et al., 2007). The approach of creating and testing defined gene deletions ultimately aids in reducing the pathogenicity/virulence of the organism without affecting the immunogenicity. Such gene-deleted organisms can be used as vaccines as they retain the immunogenic features of the wild-type organism but cannot cause disease. So far, genes involved either in determining virulence or regulating key metabolic pathways of the organism ( $\mathrm{s}$ ) have been targeted for such deletions (Babu et al., 2004). This approach has been successfully used to create several live attenuated vaccine strains of bacterial pathogens that are genetically stable, safe to use and induce better protection than killed vaccines. Gene-deleted Salmonella enterica serovar typhimurium and serovar enteritidis vaccines have been licensed for use in poultry (Babu et al., 2004) and similarly, a gene-deleted Streptococcus equi vaccine has been licensed for use in horses (Meesun et al., 2007).

Bacterial vectors: In general bacterial vectors are attenuated by deletion of genes required for key metabolic processes or genes associated for virulence. Although, they are not used routinely in animals, rapid progress is being made in developing and evaluating different bacteria as vectors. For several years, BCG (Bacillus Calmette-Guerin) and Salmonella have been developed as 
vectors for delivering vaccine antigens to animals and the latter has been used for the generation of live vaccine strains for poultry. There are currently a number of other bacterial vectors being developed based on commensal microorganisms (Lactococcus, Streptococcus, Lactobacillus and Staphylococcus) or attenuated pathogenic organisms (Shigella, Bacillus, Yersinia, Vibrio, Cornebacteria and Bordetella), all of which are being evaluated for their ability to induce protective immunity (Meesun et al., 2007).

Chimeric viruses: Chimeric viruses are defined as recombinant viruses that may contain parts of two closely related viral genomes. For example, a chimeric virus could be one that contains structural genes of one viral serotype and nonstructural genes of another serotype of the same virus. Alternatively, a chimeric virus would be one that contains part of the genome from different members belonging to the same virus family. In principle, chimeric viruses display the biological characteristics of both the parent viruses (Reimann et al., 2004). One of the main advantages of this approach is that a single dose of chimeric virus delivers the complete repertoire of antigens closely resembling the pathogen (s) which can induce protective immune response against multiple viral pathogens belonging to or different serotypes of the same viral pathogen. The availability of infectious full-length complementary DNA (cDNA) clones of different RNA viruses using reverse genetics technologies has led to novel vaccine development strategies (Van Gennip et al., 2000).

Chimeric pestiviruses have been constructed using an infectious cDNA clone containing the Classical Swine Fever Virus (CSFV) genome or the Bovine Viral Diarrhoea Virus (BVDV) genome backbones. In one instance, a chimeric pestivirus was constructed by replacing the BVDV E2 coding sequence in the infectious DNA copy of BVDV strain CP7 with the correspondingm E2 coding sequence of CSFV strain Alfort 187 (Reimann et al., 2004). Another chimeric virus was constructed by replacing the CSFV E2 coding sequence in the infectious DNA copy of CSFV vaccine strain $\mathrm{C}$ with the corresponding E2 coding sequence from BVDV (Van Gennip et al., 2000). These chimeric viruses appeared to be attenuated in pigs induced complete protection against CSFV challenge and helped to discriminate between vaccinated and infected pigs (Reimann et al., 2004).

Subunit vaccine: Subunit vaccines composed of semi-pure or purified proteins have been commercially available, since, the early 1980 's with subunit components produced by recombinant DNA technology available, since, the 1990's (Cohen, 1993). The latter have attracted growing interest and activity since that time. Subunit vaccines do not include live recombinant vector technologies which provide the delivery of recombinant proteins in vivo. The field of genomics and related areas has revolutionized the manner in which microbial antigens are identified (Van Gennip et al., 2000).

Virus-like particles: Virus-Like Particles (VLPs) are supra-molecular structures composed of one or more recombinant proteins. The particles form through self-assembly and typically range from $20-100 \mathrm{~nm}$ in size. Depending on the origin they can be icosahedral or rod-like in structure (Jennings and Bachmann, 2008). VLPs offer the advantage of formulating the vaccine antigen in a particulate structure, thereby increasing the immunogenicity of the vaccine (Zuckerman, 2006). VLPs can be used as either vaccine itself or as carrier for genetically fused (chimeric) incorporated or covalently linked antigens (Jennings and Bachmann, 2008). VLPs have been extensively studied for the past 20 years with human vaccines against Hepatitis B virus and human papillomavirus commercially available and several vaccines for veterinary application in development. These include vaccine for bluetongue virus, rota and parvovirus (Zuckerman, 2006).

DNA vaccine: DNA vaccines can be defined as antigen-encoding bacterial plasmids that are capable of inducing specific immune responses upon inoculation into a suitable host (Kim et al., 2001). DNA vaccines are the third generation vaccines based on purified plasmid preparations containing transgenes that encode antigenic/therapeutic proteins or peptides capable of triggering an immune response against a wide range of diseases (Pereira et al., 2014). Immunization is accomplished by the uptake of purified plasmid in the host cells where it persists extra-chromosomally in the nuclei. This vaccine platform presents several attributes that confer distinct advantages over other vaccine technologies in terms of safety, ease of fabrication and stability. One of the greatest advantages is the ability of DNA vaccines to induce both humoral and cell-mediated immune responses which is critical for protection from many diseases. There is also evidence that DNA vaccines can induce long-term immunity which is a further requirement for vaccine efficacy (Pereira et al., 2014).

As the vector itself does not induce immune responses, DNA vaccines can be repeatedly administered without the interference of antibodies. From a technical viewpoint, DNA vaccines are easy to engineer, produce and purify, so, new DNA vaccines can be constructed 
and evaluated in animal models within months. DNA vaccines are very stable and therefore have a long shelf life and can be transported without a cold chain. The safety of DNA vaccines has been established in various trials in several species including humans (Kim et al., 2001). Many aspects such as antigen expression and especially vector design are under study because of their great influence on immunogenicity and efficacy of DNA vaccines (Pereira et al., 2014).

Environmental application of recombinant DNA technology: Genetic engineering has wide applications in solving the environmental issues (Khan et al., 2016). The release of genetically engineered microbes, for example, Pseudomonas fluorescens strain designated HK44 for bioremediation purposes in the field was first practiced by University of Tennessee and Oak Ridge National Laboratory by working in collaboration (Ripp et al., 2000). The engineered strain contained naphthalene catabolic plasmid pUTK21 and a transposon-based bioluminescence-producing $l u x$ gene fused within a promoter that resulted in improved naphthalene degradation and a coincident bioluminescent response. HK 44 serves as a reporter for naphthalene bioavailability and biodegradation whereas its bioluminescence signaling ability makes it able to be used as an online tool for in situ monitoring of bioremediation processes. The production of bioluminescent signal is detectable using fiber optics and photon counting modules (Khan et al., 2016). Recent biotechnological approaches for bioremediation include biosorption, phytostabilization, hyperaccumulation, dendroremediation, biostimulation, mycoremediation, cyanoremediation and genoremediation which majorlydepend on enhancing or preventing specified genes activities. However, the challenges in adopting the successful technique cannot be ignored (Mani and Kumar, 2014).

\section{CONCLUSION}

Recombinant DNA technology has seven steps (isolation of gene, preparation of target DNA insertion of DNA into vector insertion of plasmid back into cell, plasmid multiplication, Target Cell Production and Cells Produced Proteins) techniques. Two enzymes (restriction endonuclease and DNA ligase) play vital role in recombinant DNA technology. Recombinant DNA technology has great roles in agriculture: manufacturing of novel enzymes for food processing, microbial strains production for industrial use, production of good quality and high yielding plants and animals. In medicine, recombinant DNA technology used for gene therapy, hormone production, antibody production and vaccine production. Concerning to environment, recombinant DNA technology involved in bioremediation.

\section{RECOMMENDATIONS}

Based on the above conclusions, the following recommendations forwarded. Agricultural, medical and environmental sectors should work cooperatively to extend and internalize recombinant DNA technology widely. Further, researches should be done to simplify tedious recombinant DNA technique procedures. Agricultural, medical and environmental sectors should work on societal perceptions, train professionals and introduce its uses to the society.

\section{ABBERVIATIONS}

\begin{tabular}{|c|c|}
\hline BVDV & $=$ Bovine Viral Diarrhoea Virus \\
\hline ADA-SCID & $=$ Adenosine Deaminase-deficiency and \\
\hline & Severe Combined Immuno Deficiency \\
\hline AIDS & $=$ Acquired Immuno Deficiency Syndrome \\
\hline ATP & $=$ Adenine Triposphate \\
\hline $\mathrm{BACS}$ & $=$ Bacterial Artificial Chromosomes \\
\hline cDNA & $=$ Complementary DNA \\
\hline $\mathrm{CHO}$ & $=$ Chinese Hamster Ovary \\
\hline CSFV & $=$ Classical Swine Fever Virus \\
\hline DNA & $=$ Deoxyribonucleic Acid \\
\hline HIV & $=$ Human Immunodeficiency Virus \\
\hline PCR & $=$ Polymerase Chain Reaction \\
\hline PSE & $=$ Pale Soft Exudate \\
\hline pST & $=$ Porcine somatotropin \\
\hline PSTOL1 & $=$ Phosphorus Starvationtolerance One (1) \\
\hline QTL & $=$ Quantitative Trait Locus \\
\hline ST & $=$ Somatotropin \\
\hline VLPs & $=$ Virus-Like Particles \\
\hline YACS & $=$ Yeast Artificial Chromosomes \\
\hline WHO & $=$ World Health Organization \\
\hline$\lambda$ & $=$ Lambda \\
\hline
\end{tabular}

\section{REFERENCES}

Aiuti, A., S. Vai, A. Mortellaro, G. Casorati and F. Ficara et al., 2002. Immune reconstitution in ADA-SCID after PBL gene therapy and discontinuation of enzyme replacement. Nat. Med., 8: 423-425.

Anonymous, 2018. Constitution of WHO: Principles. World Health Organization (WHO), Geneva, Switzerland. https:/www.who.int/about/mission/en/ 
Babu, U., R.A. Dalloul, M. Okamura, H.S. Lillehoj and H. Xie et al., 2004. Salmonella enteritidis clearance and immune responses in chickens following Salmonella vaccination and challenge. Vet. Immunol. Immunopathol., 101: 251-257.

Brophy, B., G. Smolenski, T. Wheeler, D. Wells, P. L'Huillier and G. Laible, 2003. Cloned transgenic cattle produce milk with higher levels of $\beta$-casein and K-casein. Nat. Biotechnol., 21: 157-162.

Brown, T.A., 2006. Gene Cloning and DNA Analysis: An Introduction. 5th Edn., Wiley-Blackwell Company, Hoboken, New Jersey, USA., Pages: 386.

Cardi, T. and C.N. Stewart Jr, 2016. Progress of targeted genome modification approaches in higher plants. Plant Cell Rep., 35: 1401-1416.

Cohen, J., 1993. Naked DNA points way to vaccines. Sci., 259: 1691-1693.

Galambos, L. and J.L. Sturchio, 1998. Pharmaceutical firms and the transition to biotechnology: A study in strategic innovation. Bus. Hist. Rev., 72: 250-278.

Gerdts, V., G.K. Mutwiri, S.K. Tikoo and L.A. Babiuk, 2006. Mucosal delivery of vaccines in domestic animals. Vet. Res., 37: 487-510.

Ginn, S.L., I.E. Alexander, M.L. Edelstein, M.R. Abedi and J. Wixon, 2013. Gene therapy clinical trials worldwide to 2012-an update. J. Gene Med., 15: 65-77.

Gupta, S. and C.V. Savalia, 2012. Application of biotechnology to improve livestock products. Vet. World, 5: 634-638.

Ionescu, R.M., J. Vlasak, C. Price and M. Kirchmeier, 2008. Contribution of variable domains to the stability of humanized IgG1 monoclonal antibodies. J. Pharm. Sci., 97: 1414-1426.

Jennings, G.T. and M.F. Bachmann, 2008. The coming of age of virus-like particle vaccines. Biol. Chem., 389: 521-536.

Kahi, A.K. and T.O. Rewe, 2008. Biotechnology in livestock production: Overview of possibilities for Africa. Afr. J. Biotechnol., 7: 4984-4991.

Khan, S., M.W. Ullah, R. Siddique, G. Nabi and S. Manan et al., 2016. Role of recombinant DNA technology to improve life. Intl. J. Genomics, 2016: 1-14.

Kim, J.J., J.S. Yang, L.K. Nottingham, W. Tang and $\mathrm{K}$. Dang et al., 2001. Induction of immune responses and safety profiles in rhesus macaques immunized with a DNA vaccine expressing human prostate specific antigen. Oncogene, 20: 4497-4506.

Kumar, S. and A. Kumar, 2015. Role of genetic engineering in agriculture. Plant Arch., 15: 1-6.

Kwaga, J.K.P. and J. Kabir, 1999. Basic principles of Genetic engineering and applications in Veterinary medicine. Nig. Vet. J., 20: 17-33.
Liu, W., J.S. Yuan and C.N. Stewart Jr, 2013. Advanced genetic tools for plant biotechnology. Nat. Rev. Genetics, 14: 781-793.

Lomedico, P.T., 1982. Use of recombinant DNA technology to program eukaryotic cells to synthesize rat Proinsulin: A rapid expression assay for cloned genes. Proc. National Acad. Sci., 79: 5798-5802.

Ma, J.K.C., P.M.W. Drake and P. Christou, 2003. The production of recombinant pharmaceutical proteins in plants. Nat. Rev. Genet., 4: 794-805.

Mandrich, L., 2013. Cloning and transgenesis. Rev. Art., 2: $1-4$.

Mani, D. and C. Kumar, 2014. Biotechnological advances in bioremediation of heavy metals contaminated ecosystems: An overview with special reference to phytoremediation. Intl. J. Environ. Sci. Technol., 11: 843-872.

Meeusen, E.N., J. Walker, A. Peters, P.P. Pastoret and G. Jungersen, 2007. Current status of veterinary vaccines. Clin. Microbiol. Rev., 20: 489-510.

Oikawa, T., H. Maeda, T. Oguchi, T. Yamaguchi and N. Tanabe et al., 2015. The birth of a black rice gene and its local spread by introgression. Plant Cell, 27: 2401-2414.

Olempska-Beer, Z.S., R.I. Merker, M.D. Ditto and M.J. DiNovi, 2006. Food-processing enzymes from recombinant microorganisms: A review. Regul. Toxicol. Pharmacol., 45: 144-158.

Pereira, V.B., M. Zurita-Turk, T.D.L. Saraiva, C.P. de Castro and B.M. Souza et al., 2014. DNA vaccines approach: From concepts to applications. World J. Vaccines, 4: 50-71.

Pursel, V.G., R.J. Wall, A.D. Mitchell, T.H. Elsasser and M.B. Solomon et al., 1999. Expression of Insulin-Like Growth Factor-I in Skeletal Muscle of Transgenic Swine. In: Transgenic Animals in Agriculture, Murray, J.D. (Ed.). Centre for Agriculture and Bioscience International, Wallingford, Oxfordshire, UK., ISBN:9780851992938, pp: 131-144.

Raghavan, V. and S.L. Goswami, 2007. Transgenic farm animals-a mobile pharmaceutical industry. Indian Dairyman, 59: 26-32.

Rajakaruna, S.S. and A.W. Taylor-Robinson, 2016. Application of recombinant DNA technology (Genetically Modified Organisms) to the advancement of agriculture, medicine, bioremediation and biotechnology industries. J. Appl. Biotechnol. Bioeng., 1: 78-80.

Reimann, I., K. Depner, S. Trapp and M. Beer, 2004. An avirulent chimeric Pestivirus with altered cell tropism protects pigs against lethal infection with classical swine fever virus. Virology, 322: 143-157. 
Ripp, S., D.E. Nivens, Y. Ahn, C. Werner and J. Jarrell IV et al., 2000. Controlled field release of a bioluminescent genetically engineered microorganism for bioremediation process monitoring and control. Environ. Sci. Technol., 34: 846-853.

Rostami-Hodjegan, A. and G.T. Tucker, 2007. Simulation and prediction of In vivo drug metabolism in human populations from In vitro data. Nat. Rev. Drug Discovery, 6: 140-148.

Shivanand, P. and S. Noopur, 2010. Recombinant DNA technology and genetic engineering: $\mathrm{A}$ safe and effective meaning for production valuable biologicals. Intl. J. Pharm. Sci. Rev. Res., 1: 14-20.

Soetan, K.O. and M.O. Abatan, 2008. Biotechnology a key tool to breakthrough in medical and veterinary research. Biotechnol. Mol. Biol. Rev., 3: 88-94.

Stoger, E., C. Vaquero, E. Torres, M. Sack and L. Nicholson et al., 2000. Cereal crops as viable production and storage systems for pharmaceutical scFv antibodies. Plant Mol. Biol., 42: 583-590.

Stryjewska, A., K. Kiepura, T. Librowski and S. Lochynski, 2013. Biotechnology and genetic engineering in the new drug development: Part I: DNA technology and recombinant proteins. Pharmacol. Rep., 65: 1075-1085.
Thallinger, B., E.N. Prasetyo, G.S. Nyanhongo and G.M. Guebitz, 2013. Antimicrobial enzymes: An emerging strategy to fight microbes and microbial biofilms. Biotechnol. J., 8: 97-109.

Ullah, M.W., W.A. Khattak, M. Ul-Islam, S. Khan and J.K. Park, 2015. Encapsulated yeast cell-free system: A strategy for cost-effective and sustainable production of bio-ethanol in consecutive batches. Biotechnol. Bioprocess Eng., 20: 561-575.

Van Gennip, H.G.P., P.A. van Rijn, M.N. Widjojoatmodjo, A.J. de Smit and R.J.M. Moormann, 2000. Chimeric classical swine fever viruses containing envelope protein ERNS or E2 of bovine viral diarrhoea virus protect pigs against challenge with CSFV and induce a distinguishable antibody response. Vaccine, 19: 447-459.

Zhang, J., E.B. Tarbet, H. Toro and D.C.C. Tang, 2011. Adenovirus-vectored drug-vaccine duo as a potential driver for conferring mass protection against infectious diseases. Expert Rev. Vaccines, 10: 1539-1552.

Zuckerman, J.N., 2006. Vaccination against Hepatitis A and B: Developments, deployment and delusions. Curr. Opin. Infect. Dis., 19: 456-459. 